\title{
Teaching of major communicable diseases in Sudanese medical schools: a critical look
}

\author{
S.H. Abdelrahman, ${ }^{7}$ A.M. Malik, ${ }^{2}$ A.A. Mohamedani, ${ }^{3}$ M.E. Mohamed, ${ }^{1}$ S.M. Alfadil, ${ }^{4}$ T.D.A. Elhasan ${ }^{5}$ and H.S. Ahmed ${ }^{2}$
}

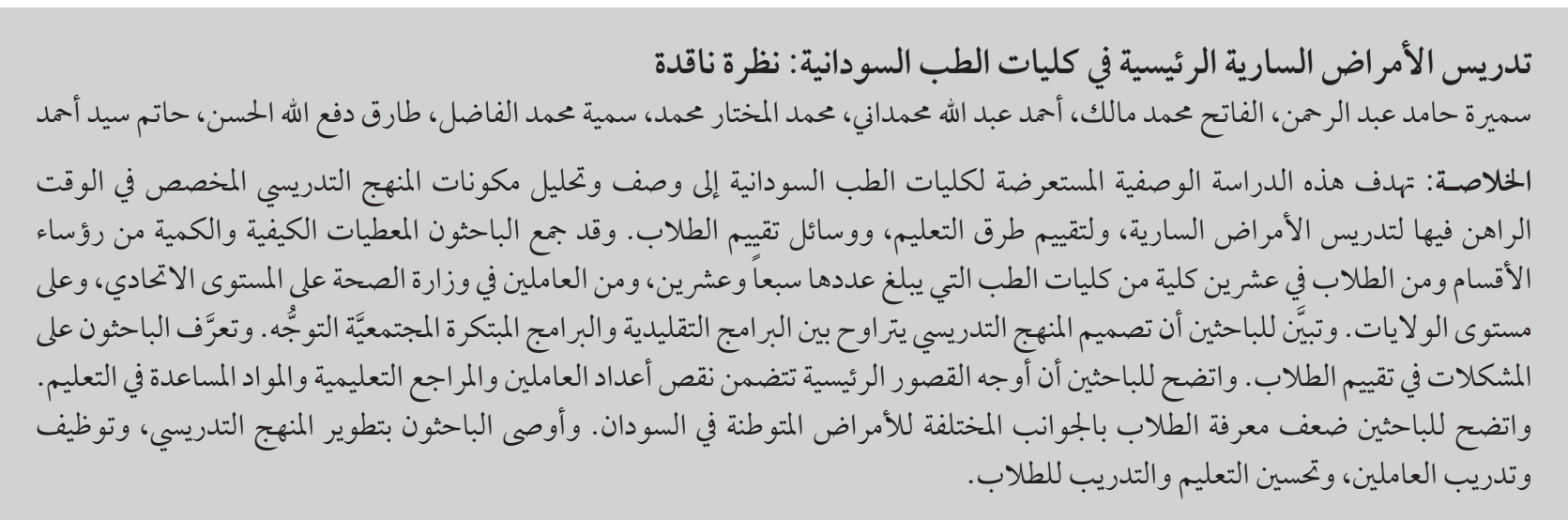

ABSTRACT This descriptive, cross-sectional study of Sudanese medical schools aimed to describe and analyse the proportion of their curricula currently allocated for teaching of communicable diseases and to assess the teaching methods and student assessment tools. Qualitative and quantitative data were collected from heads of departments and students in 20 of the 27 medical faculties and from ministry of health staff at federal and state levels. Curriculum designs ranged from traditional to innovative, community-oriented programmes. Problems regarding student evaluations were identified. Major limitations included shortages of staff, reference materials and teaching aids. Poor knowledge of students about different aspects of diseases endemic in Sudan was found. Recommendations include curriculum development, staff recruitment and training, and improvement of teaching and training of students.

Regard critique sur l'enseignement des principales maladies transmissibles dans les facultés de médecine soudanaises

RÉSUMÉ La présente étude descriptive et transversale des facultés de médecine soudanaises a tenté de décrire et d'évaluer la part de leurs programmes actuellement consacrée à l'enseignement des maladies transmissibles, et d'analyser les méthodes d'enseignement et les outils d'évaluation des étudiants. Des données qualitatives et quantitatives ont été recueillies auprès des responsables de départements et des étudiants dans 20 facultés de médecine (sur les 27 existantes), et auprès du personnel des ministères de la Santé au niveau de la fédération et des États. La structure des programmes était soit traditionnelle, soit innovante avec une orientation communautaire. Des difficultés ont été identifiées au sujet des évaluations des étudiants. Le manque de personnel, de documents de référence et de matériel pédagogique comptent parmi les freins à l'enseignement les plus importants. L'étude a révélé que les étudiants avaient des connaissances médiocres sur les différents aspects des maladies endémiques au Soudan. Les recommandations portent sur l'élaboration de programmes, le recrutement et la formation de personnel et l'amélioration de l'enseignement dispensé aux étudiants.

${ }^{7}$ Department of Community Medicine; ${ }^{3}$ Department of Pathology, Faculty of Medicine, University of Gezira, Wad Madani, Sudan (Correspondence to S.H.Abdelrahman:samhamid2002@yahoo.co.uk).

${ }^{2}$ Federal Ministry of Health, Khartoum, Sudan. ${ }^{4}$ World Health Organization Country Office, Khartoum, Sudan. ${ }^{5}$ Gezira State Ministry of Health, Wad Madani, Sudan

Received: 15/04/10; accepted: 23/08/10 


\section{Introduction}

The First International Conference on Primary Health Care in Alma-Ata in 1978 confirmed the need for a new look at the education of health professionals. This global perspective on the world's health problems and, subsequently, the promotion of the target of health for all by the year 2000 brought with it a growing recognition the need to adjust health manpower development to the real health needs and demands of the community [1]. This new focus on medical education that is relevant to community health needs means that the subject matter studied by medical students should have direct relevance to the priority health problems of the society for which these students are trained [2].

Communicable diseases dominate the health scene in Sudan, with its high vulnerability to outbreaks of malaria, schistosomiasis, tuberculosis (TB), leishmaniasis, leprosy, cholera and many others. Despite a major expansion in higher education in Sudan since 1993, especially in the area of medical education, unpublished examination reports from some medical schools suggest that a high proportion of medical students lack the clinical skills and other competencies necessary for the prevention, control and management of major endemic diseases in Sudan. There is also concern about the coordination and partnership between medical schools and relevant programmes of the Sudan federal ministry of health $(\mathrm{MOH})$ in designing and implementing the necessary educational programmes.

The main objective of the study was to describe and identify the problems and limitations of the current teaching of major endemic diseases in Sudan in the curricula of Sudanese medical schools and to propose recommendations for strengthening and further development of education in this area.

\section{Methods}

The study was conducted in 2007-08 and used a descriptive design in which both qualitative and quantitative instruments were used to fulfil the study objectives.

\section{Sample}

The sampling frame consisted of all Sudanese medical faculties $(n=27)$ in all 4 regions of the country and the capital city Khartoum. In order to guard against confounding factors related to recently established medical schools we included only medical schools that had graduated at least 2 batches of graduates. Out of the 23 medical schools that satisfied the inclusion criteria, 20 were included in the study; 3 schools were not investigated due to security problems or a student strike that lead to closure of the university concerned. The distribution of the study medical faculties in the different regions of Sudan is shown in Table 1.

\section{Data collection}

A total of 14 forms were designed for the different stages of data collection. Data was collected by 6 team members, comprising academic staff of the universities and staff from the federal $\mathrm{MOH}$, all of whom were $\mathrm{PhD}$ holders with a rich experience in similar research. The data collection team met all the deans of faculties to explain the study objectives and its expected outcomes and to conduct structured interviews with the heads of the departments of medicine, community medicine, pathology and paediatrics.

Data were collected on: background information about the medical school; the curriculum content concerning diseases endemic in Sudan; the proportion of the overall curriculum dedicated to teaching endemic diseases (contact hours); the educational methods used; and the availability of reference materials (books, training and learning materials, Internet resources).
Written assessments were done on a sample of students who had already completed all courses related to endemic diseases. The total number of eligible students at each school was provided by the faculty registrar and 10\% of them were randomly selected, with proportionate representation of males and females. The total number of students who agreed to participate in the assessment was 290. The assessments covered knowledge about 24 major diseases that are endemic in Sudan in the following areas: disease epidemiology (causative agent(s), vector, reservoir, route(s) of transmission); laboratory diagnosis; and federal $\mathrm{MOH}$ strategies and protocols for treatment, prevention and control of those diseases [3-11].

To collect qualitative data about the teaching of endemic diseases and how to improve it, focus group discussions (FGDs) were conducted with students at the medical faculties. One FGD was conducted in each medical school (20 groups in total), each group consisting of 8-10 students (males and females). FGDs were also carried out with managers of disease programmes $(n=6)$ at the federal $\mathrm{MOH}$ and in-depth interviews were conducted with the directors general $(n=12)$ at the $\mathrm{MOH}$ in the states where the investigated faculties were located.

For analysis the curricula of the medical schools were categorized into 3 main types: innovative (communityoriented), traditional or hybrid (mixture of the 2 types).

\section{Data analysis}

SPSS, version 7.5 was used to analyse quantitative data. Descriptive statistics was also used to quantify the existence of different types of educational resources. Qualitative data was analysed manually.

\section{Results}

The length of the academic programme was 5 years in $7(35 \%)$ of the medical 


\begin{tabular}{|c|c|c|c|c|c|c|}
\hline \multirow[t]{2}{*}{ Type of medical school } & \multicolumn{6}{|c|}{ No. of faculties } \\
\hline & Northern & Eastern & Central & Western & Southern & Khartoum \\
\hline Public & 3 & 3 & 4 & 1 & 2 & 4 \\
\hline Private & 0 & 0 & 0 & 0 & 0 & 3 \\
\hline Total & 3 & 3 & 4 & 1 & 2 & 7 \\
\hline
\end{tabular}

faculties and 6 years in 13 (65\%). Student intake per batch varied considerably between the faculties, ranging from $95-250$. It was generally larger in government-run medical faculties than private ones.

\section{Review of curriculum and teaching in medical faculties}

\section{Curriculum}

The curriculum was documented in $18(90 \%)$ of the faculties and the documents of different courses were available to faculty staff in 16 of these. Curriculum handouts were distributed to students in 9 of the faculties (45\%). As reported by the faculties, 17 of them had an existing curriculum review committee. However, a curriculum review had been conducted in only 9 faculties: 13 years ago in 1 faculty, 3 years ago in 5 faculties, 2 years ago in 1 faculty, 1 year ago in 2 faculties. Students were represented in the review committees of only 7 (35\%) faculties. In 10 faculties (50\%), staff from the federal $\mathrm{MOH}$ had participated in the initial development of the faculty curriculum and in 5 medical schools (25\%) there had been participation by state $\mathrm{MOH}$ staff.

\section{Staff}

Considering both full- and part-time staff, the range of staff:student ratios in the 4 studied departments in the different medical faculties was as follows:

- Medicine: 1:10 (7 faculties), 1:111:15 (10 faculties), > 1:15 (3 faculties);

- Pathology: 1:15-1:20 (8 faculties), 1:21-1:30 (8 faculties), 1:31-1:70 (4 faculties);
- Paediatrics: 1:10 (7 faculties), 1:111:15 (4 faculties), 1:16-1:30 (9 faculties);

- Community medicine: 1:11-1:15 (4 faculties), 1:16-1:30 (10 faculties), 1:40 (2 faculties), 1:50 (2 faculties), $1: 100$ ( 2 faculties).

In 3 of the faculties (15\%) a proportion of the faculty staff (mainly from the departments of medicine, paediatrics, pathology and community medicine) were also affiliated to the state $\mathrm{MOH}$. Participation of faculty staff in $\mathrm{MOH}$ activities addressing endemic diseases are shown in Table 2. The most common activity was training of health workers by staff in $60 \%$ of faculties.

Only 4 faculties (20\%) had set plans for regular staff orientation and updating in the different aspects of endemic diseases. Technical updates regarding endemic diseases were received from the federal MOH by only 10 (50\%) of the faculties. In 4 of these faculties the updates were disseminated to faculty staff on a personal basis only, with no mechanism identified for dissemination to ensure coverage of all relevant departments if not all faculty staff. The received updates were mainly related to TB (DOTS), diarrhoeal diseases, polio eradication and malaria protocols for the treatment of cases.

\section{Teaching}

The type of curriculum was classified as traditional in 7 medical schools, innovative in 8 and hybrid in 5 . Of the faculties 11 (55\%) had discipline-based systems and 9 (45\%) adopted the integrated, system-based block system; 5 of the latter group followed a problem-solving approach. Theoretical and clinical training about endemic diseases were implemented in teaching hospitals in all the faculties, whereas training in a primary health care setting was conducted in only $5(25 \%)$ of the faculties.

The total contact hours allocated for the teaching of endemic diseases in the curriculum varied between 6368-7310, credit hours from 180-271 and the number of weeks from 140-180.

\section{Coverage of endemic diseases in the curriculum}

Table 3 shows the coverage of the 24 major endemic diseases in the medical faculty curricula. Only 11 of the diseases

\begin{tabular}{lcc}
\hline $\begin{array}{l}\text { Table 2 Participation of medical faculty staff in Ministry of Health activities } \\
\text { faculties) }\end{array}$ & No. of faculties \\
\hline Aspect of staff participation & 12 & $\%$ \\
Training of health workers & 8 & 60 \\
Setting priorities & 8 & 40 \\
Treatment protocols & 7 & 40 \\
Guidelines for diagnosis & 6 & 35 \\
Preparation of training packages & 6 & 30 \\
Monitoring and evaluation & 5 & 30 \\
Research projects & 4 & 25 \\
Guidelines for control and prevention & 4 & 20 \\
Development of communication plans & & 20 \\
\hline
\end{tabular}




\begin{tabular}{|c|c|c|c|c|c|c|c|c|c|}
\hline \multirow[t]{3}{*}{ Disease } & \multirow{3}{*}{$\begin{array}{c}\text { No. of } \\
\text { faculties } \\
\text { teaching } \\
\text { disease }\end{array}$} & \multicolumn{8}{|c|}{ Faculties with excellent to moderate coverage of: } \\
\hline & & \multicolumn{2}{|c|}{ Epidemiology } & \multicolumn{2}{|c|}{ Diagnosis } & \multicolumn{2}{|c|}{ Management } & \multicolumn{2}{|c|}{$\begin{array}{l}\text { Control \& } \\
\text { prevention }\end{array}$} \\
\hline & & No. & $\%$ & No. & $\%$ & No. & $\%$ & No. & $\%$ \\
\hline Malaria & 20 & 18 & 90 & 18 & 90 & 18 & 90 & 18 & 90 \\
\hline Schistosomiasis & 20 & 18 & 90 & 18 & 90 & 18 & 90 & 18 & 90 \\
\hline Leishmaniasis & 20 & 18 & 90 & 19 & 95 & 18 & 90 & 18 & 90 \\
\hline Tuberculosis & 20 & 18 & 90 & 19 & 95 & 18 & 90 & 18 & 90 \\
\hline Diarrhoeal diseases & 20 & 16 & 80 & 18 & 90 & 18 & 90 & 16 & 80 \\
\hline Cholera & 20 & 16 & 80 & 18 & 90 & 18 & 90 & 16 & 80 \\
\hline Typhoid & 20 & 14 & 70 & 18 & 90 & 18 & 90 & 18 & 90 \\
\hline Brucellosis & 20 & 16 & 80 & 19 & 95 & 18 & 90 & 18 & 90 \\
\hline Hepatitides & 20 & 18 & 90 & 19 & 95 & 18 & 90 & 18 & 90 \\
\hline Meningitis & 20 & 18 & 90 & 19 & 95 & 18 & 90 & 18 & 90 \\
\hline Diphtheria & 20 & 16 & 80 & 16 & 80 & 16 & 80 & 16 & 80 \\
\hline AIDS & 19 & 15 & 79 & 17 & 89 & 17 & 89 & 17 & 89 \\
\hline Leprosy & 18 & 14 & 78 & 16 & 89 & 16 & 89 & 14 & 78 \\
\hline Tetanus & 18 & 16 & 89 & 16 & 89 & 16 & 89 & 14 & 78 \\
\hline Whooping cough & 18 & 14 & 78 & 14 & 78 & 14 & 78 & 14 & 78 \\
\hline Measles & 18 & 14 & 78 & 14 & 78 & 14 & 78 & 14 & 78 \\
\hline Trachoma & 17 & 6 & 35 & 6 & 35 & 7 & 41 & 6 & 35 \\
\hline Scabies & 17 & 4 & 24 & 6 & 35 & 6 & 35 & 6 & 35 \\
\hline Intestinal worms & 16 & 12 & 75 & 14 & 88 & 14 & 88 & 12 & 75 \\
\hline Filariasis & 15 & 8 & 53 & 10 & 67 & 8 & 53 & 8 & 53 \\
\hline Haemorrhagic fevers & 15 & 7 & 47 & 3 & 20 & 8 & 53 & 3 & 20 \\
\hline Trypanosomiasis & 15 & 5 & 33 & 7 & 47 & 5 & 33 & 5 & 33 \\
\hline Poliomyelitis & 15 & 13 & 87 & 13 & 87 & 13 & 87 & 12 & 80 \\
\hline Anthrax & 9 & 1 & 11 & 1 & 11 & 2 & 22 & 1 & 11 \\
\hline
\end{tabular}

assessed were covered by all 20 faculties and only 9 of the medical schools (45\%) addressed all 24 diseases. Important endemic diseases such as poliomyelitis, tetanus, leishmaniasis and AIDS were not covered by some faculties. Leprosy and trypanosomiasis were also neglected. Anthrax were addressed by 11 of the faculties.

Each disease was also analysed by 4 content areas (epidemiology, diagnosis, management, prevention and control) with each area further divided into subareas. Faculties that covered all major areas and all identified subareas were considered to have excellent coverage, while the schools whose curricula did not cover all 4 major areas or did not cover all subareas (but still covered 7-9 subareas) were considered to have moderate coverage (Table 3). Diseases addressed by all (100\%) of the medical schools had excellent coverage in all aspects (epidemiology, diagnosis, management, control and prevention). Moderate coverage was found in the group of diseases addressed by $90 \%-95 \%$ of the schools, while the majority of diseases that were addressed by $<85 \%$ of the schools had deficient coverage of these aspects.

In Table 4 medical faculties were further described by their type of curriculum (traditional, innovative or hybrid). Epidemiology, diagnosis, management and prevention and control of malaria, schistosomiasis, leishmaniasis,
$\mathrm{TB}$ and the hepatitides were addressed in the curriculum by $80 \%$ or more of medical faculties of all 3 curriculum types. Diseases that were covered by less than $80 \%$ of medical schools in all 3 curriculum types included: trachoma, filariasis, trypanosomiasis, scabies and anthrax. Haemorrhagic fevers were neglected in some areas, but diagnosis was addressed by more than $80 \%$ of the traditional and innovative faculties. The percentage of faculties addressing the specified endemic diseases fell below $40 \%$ for scabies and anthrax for each of the 3 curriculum types. For the remaining list of diseases, the table shows clear variations between the 3 categories of medical faculties with regard to the teaching in the 4 main areas. 


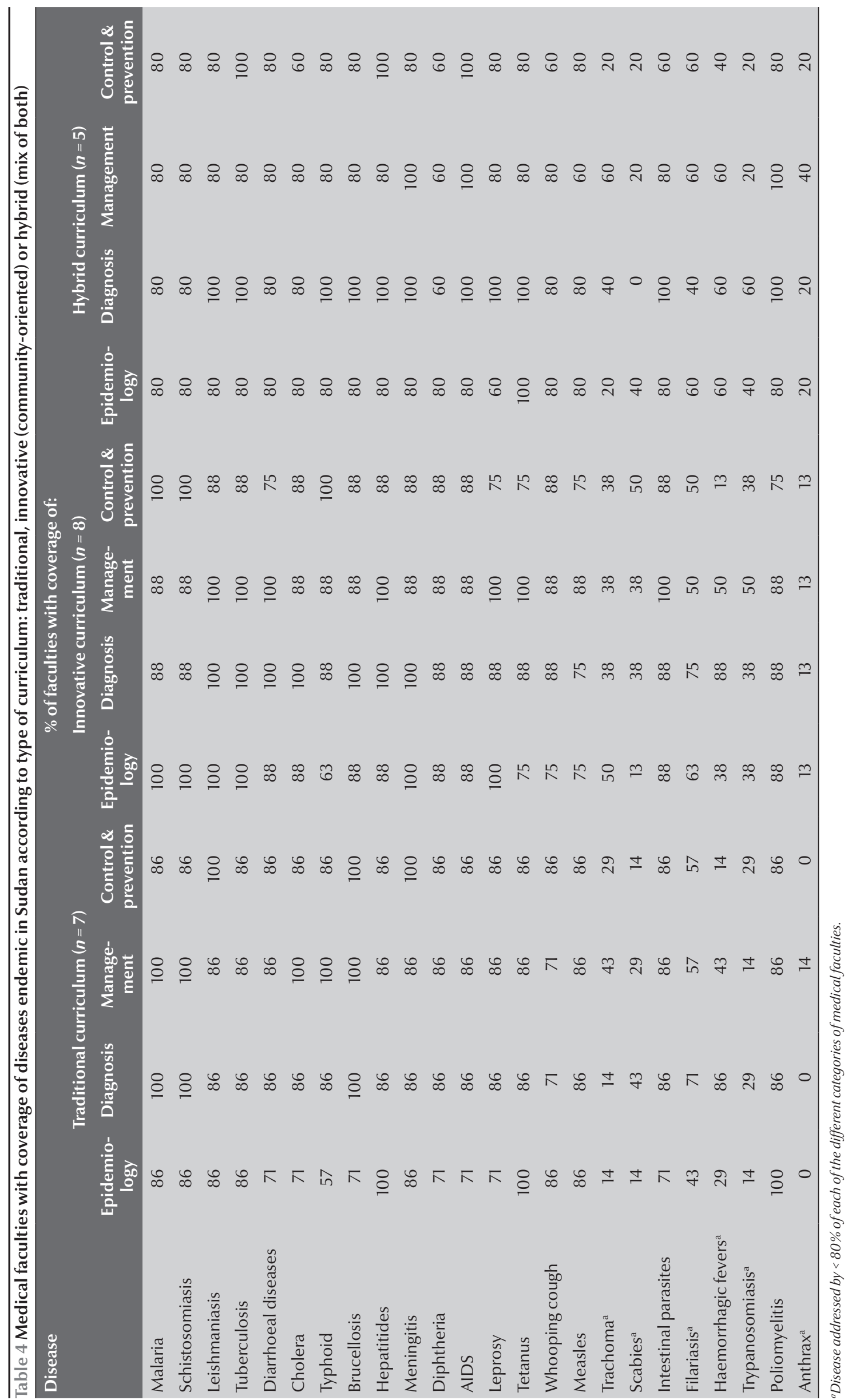




\section{Contact hours allocated for teaching of endemic diseases}

Around 107-276 total contact hours for the curriculum were allocated for the teaching of endemic diseases in the different faculties.

- Theoretical teaching: contact hours per subject ranged from $1-22$. The lowest coverage was for anthrax ( $50 \%$ of faculties). Scabies, haemorrhagic fevers, trypanosomiasis, cholera, filariasis, leprosy and diarrhoeal diseases were covered by between $70 \%-90 \%$ of the faculties. The remaining diseases were covered by $100 \%$ of faculties.

- Practical training: contact hours per subject ranged from $0-9$. None of the faculties $(0 \%)$ had practical training for 9 subjects (AIDS, trachoma, haemorrhagic fevers, polio, diphtheria, tetanus, whooping cough, scabies and anthrax). Practical training was done only for the major diseases that were covered by $100 \%$ of schools.

- Field training: contact hours per subject ranged from 1-3. There was no field training for 11 subjects (AIDS, trachoma, haemorrhagic fevers, filariasis, polio, diphtheria, tetanus, whooping cough, scabies, measles and anthrax) not covered by any type of field training. Field training was done for the major diseases that were covered by $100 \%$ of schools.

- Clinical training: contact hours per subject ranged from 1-4. All faculties $(100 \%)$ covered malaria, TB, diarrhoeal diseases, meningitis and hepatitides.

\section{Use of $\mathrm{MOH}$ protocols on endemic diseases}

The utilization by the medical faculties of federal $\mathrm{MOH}$ strategies, guidelines and protocols in diagnosis, treatment, prevention and control of endemic diseases was very variable. The guidelines on treatment were used by 16 faculties, on diagnosis by 13 and on prevention and control by only 2 faculties

\section{Availability of teaching materials}

Regarding the availability of reference books and some other learning aids, a minimum acceptable standard was the availability of a well-known textbook of endemic diseases and other directly related disciplines, such as microbiology, parasitology, medical entomology, etc.). Less than $25 \%$ of the faculties, both government and private, had all the references available. The US Centers for Disease Control and Prevention's Control of Communicable Diseases Manual, which is essential for teaching of endemic diseases, was available in only 7 (35\%) of the faculties. There is no standard textbook for microbiology, but there were diverse types of reference materials for parasitology, bacteriology and virology available at all faculties. Other related books on medicine, paediatrics and general pathology were available in all of the faculties. The best student/ book ratio was 4 students/book and the worst was 56 students/book, with an average ratio of 10 students/book.

Concerning the other teaching aids (e.g. slides, museum specimens, CDROMs and information, education and communication materials) all faculties had at least 1 of them available, especially the older established faculties that had museums with pathology specimens of endemic diseases. However, the availability of all these aids was zero. CDROMs about different aspects of endemic diseases were available in $7(35 \%)$ of the faculties, while video films were already obsolete at university libraries. Only 1 faculty among the studied group provided posters on endemic diseases at the library.

Museum specimens for the study of endemic diseases were available in only $20 \%$ of the faculties, and Internet services were available in $65 \%$.

\section{Instructional methods}

Lectures were the main method for theory-based instruction, utilized by all $(100 \%)$ of the faculties, while some faculties used seminars and tutorials
(40\%), problem-based learning (10\%) and a few faculties used the case-study approach (10\%). Practical laboratory training and slide demonstrations were used in only $10 \%$ and $40 \%$ of faculties respectively. Field training was done as follows: in villages ( $15 \%$ of faculties), health centres (40\%) and rural hospitals (15\%). Visits to disease programme administrations to study managerial aspects were done by a few faculties (10\%). Clinical training in teaching hospitals was done by all faculties (100\%) and in outpatient departments by only $20 \%$.

\section{Assessment methods}

Assessment of students was done through a range of methods. Long and short essays, multiple choice questions (true/false) and clinical examinations were used by all faculties (100\%); other used case scenarios $(20 \%)$, clinical problems (15\%), practical examinations (10\%), objective structured clini$\mathrm{cal} /$ practical examinations $(10 \%)$ and students' seminars (15\%).

\section{Opinions of students and programme managers}

\section{Students}

In the FGDs with students it was clear that not all the studied diseases were covered by the curriculum. Students mentioned that diseases covered to a large extent included malaria, TB, schistosomiasis, meningitis and poliomyelitis. Some diseases, such as diarrhoeal diseases, leishmaniasis, typhoid, hepatitides, leprosy and other childhood vaccine-preventable infectious diseases, were moderately covered. Poorly covered diseases (or even not covered at all) includes AIDS, filariasis, trachoma, scabies, anthrax, trypanosomiasis, cholera, haemorrhagic fevers and intestinal worms.

Students mentioned that their training on endemic diseases lacked practical and field sessions, and even clinical training was inadequate, as students had no chance to see the clinical 
presentation(s) of some diseases even in slides or photographs (e.g. filariasis, haemorrhagic fevers, trypanosomiasis). Such diseases were only mentioned during the discussion of differential diagnoses of other diseases.

Students' suggestions to promote the teaching of endemic diseases included: more focus on clinical management of cases, more focus on common endemic diseases in the region around each school, adapting teaching to the situation in Sudan, content to include $\mathrm{MOH}$ protocols and guidelines and more focus on disease prevention and control measures, provision of more references and educational materials (CDROMs, slides, models, museum specimens, etc.). Students suggested that the endemic diseases curriculum should be unified across all the medical schools and called for stronger integration between departments within each faculty. They suggested that endemic diseases should be a separate course in the faculty programme.

\section{Programme managers (federal and state $\mathrm{MOH}$ )}

FGDs with $\mathrm{MOH}$ programme managers at federal and state levels indicated that they were generally not involved in the development or review of the endemic diseases curricula in medical faculties. Few of them from the federal level participated because they were mainly part-time staff in those schools. Participation in teaching by state $\mathrm{MOH}$ staff was infrequent and was mainly done on an individual basis. The teaching methods commonly used by $\mathrm{MOH}$ staff were lectures followed by group discussions. Some federal $\mathrm{MOH}$ staff participated in student evaluations, through designing examination questions on subjects they had taught, mainly through multiple choice questions, long or short essays; there was no participation in clinical or practical tests. Staff from the state $\mathrm{MOH}$ did not participate in student evaluations.
Only the malaria control programme at federal level distributed materials to medical schools including the protocol for management of malaria. Furthermore, only the malaria control unit carried out staff orientation at medical faculties as part of orientation for all doctors at federal and state levels.

Few of the programmes at federal and state levels involved any of the medical school staff in training activities and there was no binding policy at the $\mathrm{MOH}$ to involve medical school staff. No partnerships were planned between $\mathrm{MOH}$ and medical schools in research, services or training. Participation in research activities were achieved on an individual basis. All MOH staff were in favour of partnerships with medical schools to develop a unified curriculum to achieve the objectives of both $\mathrm{MOH}$ programmes and medical schools.

\section{Knowledge of medical students about endemic diseases}

The knowledge of students who had completed their study of endemic diseases within the faculty curriculum is summarized in Table 5. An appreciable number of students were not clear about what is a vector, route of infection or what a causative agent meant. Many of them were even confused between the terms vector, causative agent and reservoir. Only a few students understood the DOTS strategy for treatment of TB Few knew about rapid diagnostic techniques for laboratory diagnosis of malaria. Leprosy was considered by some students as a sexually transmitted infection (STI). There was no knowledge of the concept of notification of diseases or disease surveillance.

\section{Discussion}

This study was a huge undertaking, covering 20 medical schools in Sudan and a list of 24 diseases. Sudan is a vast country with varying geographical and socioeconomic conditions; hence it might be expected that some schools will give priority to diseases prevalent in their locality. On the other hand, they are expected to cover a full range of other diseases because graduates are likely to practise medicine in other parts of the country, in other countries in the region and internationally. The role of a medical school should include teaching, service delivery, research and advocacy as well as active participation in national programme planning, implementation and evaluation [12].

The missions and philosophies of the investigated schools were very variable. Not all of them had clear missions and objectives and some had no written curriculum. Radical changes are taking place in undergraduate medical education worldwide [12]. The general trend is towards innovative curricula with problem-based learning, integration of basic, clinical and social sciences and perhaps multidisciplinary education [12].

This study showed a number of weaknesses in the teaching of major communicable diseases in Sudanese medical schools which can be summarized as follows: small numbers of faculty staff and inadequate training for them; lack of facilities (lecture and tutorial rooms, laboratories, teaching aids, books, etc.); lack of a written curriculum in some schools or a curriculum that has never been reviewed; practical and clinical training that is inadequate or absent; lack of or inadequate coordination and partnership between the faculties and $\mathrm{MOH}$ (both at federal and state level); lack of references and teaching materials in general; and problems with student assessments and evaluations.

Given the vital role of medical schools in national efforts to control endemic diseases, it is mandatory to reform medical school curricula to cater for this role. This was clearly shown by a World Health Organization (WHO) report of 7 Asian countries in 2000. Although the study focused on enhancing the role of medical schools in control of 


\begin{tabular}{|c|c|c|c|c|c|c|c|}
\hline \multirow[t]{2}{*}{ Disease } & \multicolumn{7}{|c|}{$\%$ of students with correct answers } \\
\hline & $\begin{array}{l}\text { Causative } \\
\text { agent(s) }\end{array}$ & Vector & Reservoir & $\begin{array}{l}\text { Route(s) of } \\
\text { transmission }\end{array}$ & $\begin{array}{l}\text { Laboratory } \\
\text { diagnosis }\end{array}$ & Treatment & $\begin{array}{c}\text { Control \& } \\
\text { prevention }\end{array}$ \\
\hline Malaria & 76 & 79 & 59 & 79 & 94 & 34 & 64 \\
\hline Schistosomiasis & 67 & 63 & 47 & 57 & 69 & 38 & 63 \\
\hline Leishmaniasis & 53 & 65 & 50 & 56 & 37 & 37 & 40 \\
\hline Tuberculosis & 86 & - & 70 & 85 & 53 & 65 & 71 \\
\hline AIDS & 39 & - & 71 & 93 & 39 & 27 & 66 \\
\hline Diarrhoeal diseases & 64 & - & 50 & 78 & 69 & 45 & 67 \\
\hline Cholera & 60 & - & 56 & 80 & 44 & 43 & 45 \\
\hline Haemorrhagic fevers & 31 & 27 & - & 34 & 13 & 11 & 22 \\
\hline Typhoid fever & 73 & - & 46 & 74 & 61 & 44 & 49 \\
\hline Brucellosis & 20 & - & 51 & 57 & 33 & 22 & 43 \\
\hline Hepatitides & 74 & - & 56 & 75 & 32 & 20 & 51 \\
\hline Meningitis & 74 & - & 62 & 37 & 69 & 34 & 58 \\
\hline Filariasis & 32 & 18 & 37 & 33 & 19 & 10 & 17 \\
\hline Intestinal worms & 49 & - & 40 & 54 & 46 & 12 & 36 \\
\hline Leprosy & 60 & - & 46 & 47 & - & 30 & 22 \\
\hline Trypanosomiasis & 15 & 45 & 36 & 44 & 15 & 7 & 23 \\
\hline Trachoma & 16 & - & 26 & 21 & - & 11 & 13 \\
\hline Poliomyelitis & 55 & - & 46 & 42 & 10 & 9 & 53 \\
\hline Diphtheria & 18 & - & 35 & 37 & - & 12 & 32 \\
\hline Tetanus & 47 & - & 32 & 40 & - & 19 & 48 \\
\hline Whooping cough & 21 & - & 34 & 46 & - & 8 & 38 \\
\hline Measles & 35 & - & 35 & 39 & - & 10 & 42 \\
\hline Scabies & 30 & - & 12 & 12 & 5 & 3 & 7 \\
\hline Anthrax & 9 & - & 10 & 18 & 6 & 6 & 7 \\
\hline
\end{tabular}

- = not applicable.

STIs, HIV and TB, the findings can be generalized to other endemic diseases. The study concluded that the growing burden of these diseases called for a broad-based multisectoral response and that the role of medical schools was crucial. Urgent action was needed by medical schools to coordinate state, national and WHO programmes [13].

Although medical schools have a role to play in national disease control programmes, their participation in control programmes in Sudan has so far been inadequate. Medical academics and practitioners lack a consensus on the rationale and practices of national control programmes, largely due to an absence of information and involvement in these. National control programmes in turn, according to statements of medical school directors and programme managers, have not been successful in reaching out to medical schools and providing them with the necessary information to make useful contributions. Similar conclusions and recommendations were made by another WHO report, following an intercountry meeting on $\mathrm{TB}$ and medical schools in 2000, attended by 14 countries in the Eastern Mediterranean region with representatives from medical schools and $\mathrm{TB}$ control programmes [14].

The majority of medical schools in our study had problems regarding the teaching of endemic diseases because there was a dichotomy between national programmes and the teaching in these schools. There was inadequate utilization of the national guidelines and protocols and of $\mathrm{MOH}$ expertise in the teaching and training of medical students. Also, partnership, coordination and collaboration between $\mathrm{MOH}$ at federal and states level and medical schools in the issue of teaching and research on endemic diseases was very weak and not institutionalized in many cases. This limitation was reflected in poor training of students in $\mathrm{MOH}$ programmes, protocols and guidelines and hence the likely creation of a dichotomy between pre-service training in medical schools and medical practice thereafter.

Based on the findings of this study we recommend adoption of the policies, strategies and protocols of the federal $\mathrm{MOH}$, reviews of existing 
curricula, adoption of active teaching/ learning methods, better provision of educational and training materials and adoption of appropriate student assessment and evaluation strategies. Participation of faculty staff in formulation of national policies and disease control programmes is also very essential. Better links are needed with the Sudan
Medical Council, the national disease control programmes and the Ministry of Higher Education and Research. WHO and other organizations have an important role in the provision of technical support, monitoring and evaluation of activities related to education, training and research in collaboration with the federal $\mathrm{MOH}$.

\section{Acknowledgements}

This study was made possible through the support of the World Health Organization Regional Office for the Eastern Mediterranean, the Sudanese Federal Ministry of Health, state $\mathrm{MOH}$ involved in the study and the administration, staff and students of the medical schools.

\section{References}

1. Fulop T. Health personnel for tomorrow: the great challenge of the health for all movement, Manila, World Health Organization Regional Office for the Western Pacific. 1985 (WRP/HMD/INF.4).

2. Magzoub ME. Studies in community-based education programme implementation and student assessment at the Faculty of Medicine, University of Gezira, Sudan [thesis]. Maastricht, University of Limburg, University Printing House, 1994

3. National Malaria Control Program. National guide for treatment ofmalaria, Khartoum, Sudan, Federal Ministry of Health, 2004.

4. National Malaria Control Program. Guide for training of volunteers on malaria control. Khartoum, Sudan, Federal Ministry of Health, 2004.

5. Kurti IA, Othman HA. Guide for distribution of the treatments of bilharzias and soil-transmitted helminthiasis. Khartoum, Sudan, Federal Ministry of Health, National Program for the Control of Bilharzias and Soil-Transmitted Helminthiasis, 2004.

6. Manual for the diagnosis and treatment of leishmaniasis. Khartoum, Sudan, Federal Ministry of Health, 2004.

7. Eltayeb EM, Nageeb M. Guidelines for leishmaniasis control in Sudan. Khartoum, Sudan, Federal Ministry of Health, Directorate General of Preventive Medicine, 1994.
8. Eltayeb EM, Elzein HA. Early prepardeness and containment of health emergencies. Khartoum, Sudan, Federal Ministry of Health, Directorate General of Preventive Medicine, 2002.

9. Chin J, ed. Control of communicable diseases manual. Washington DC, American Public Health Association, 2000.

10. Guide for the control of cerebrospinal meningitis. Khartoum, Sudan, Federal Ministry of Health, General Directorate of Preventive Medicine, 2002

11. Guide to eliminate leprosy as a public health problem. Geneva, World Health Organization, 2000 (WHO/CH/1211-27).

12. Jones GL. Beyond 'tomorrow's doctor': a review of basic medical education in the UK. Annals of Tropical Pediatrics, 2005, 25:71-78.

13. Enhancing the role of medical schools in STI/HIV and TB control. New Delhi, World Health Organization Regional Office for South East Asia, 2000.

14. Report on the intercountry meeting on tuberculosis and medical schools. Cairo, World Health Organization Regional Office for the Eastern Mediterranean, 2000. 